\title{
Association between six-minute walk test parameters and the health-related quality of life in patients with pulmonary Mycobacterium avium complex disease
}

Kazuma Yagi ${ }^{1}$, Takanori Asakura ${ }^{1}$, Ho Namkoong ${ }^{1}$, Shoji Suzuki ${ }^{1}$, Takahiro Asami ${ }^{1}$, Satoshi Okamori ${ }^{1}$,

Tatsuya Kusumoto', Yohei Funatsu', Hirofumi Kamata', Tomoyasu Nishimura², Makoto Ishii',

Tomoko Betsuyaku ${ }^{1}$ and Naoki Hasegawa ${ }^{3^{*}}$ (D)

\begin{abstract}
Background: Pulmonary Mycobacterium avium complex (pMAC) disease is a chronic, slowly progressive disease. The aim of the present study was to determine the association of six-minute walk test (6MWT) parameters with pulmonary function and the health-related quality of life (HRQL) in patients with pMAC disease.

Methods: This cross-sectional study included adult patients with pMAC and was conducted at Keio University Hospital. We investigated the relationship of 6MWT parameters with clinical parameters, including pulmonary function, and HRQL, which was assessed using the 36-Item Short Form Health Survey (SF-36) and St. George's Respiratory Questionnaire (SGRQ).
\end{abstract}

Results: In total, 103 consecutive patients with pMAC participated in 6MWT (median age, 64 years; 80 women) and completed SF-36 and SGRQ. The six-minute walk distance (6MWD) showed significant negative and positive correlations with all SGRQ domain scores $[\rho=(-0.54)-(-0.32)]$ and the physical component summary (PCS) score $(\rho=0.39)$ in SF-36, respectively; the opposite was observed for the final Borg scale (FBS) score (all SGRQ scores, $\rho=0.34-0.58$; PCS score, $\rho=-0.50$ ). The distance-saturation product showed significant negative and positive correlations with all SGRQ scores $[\rho=(-0.29)-(-0.55)]$ and the PCS score $(\rho=0.40)$, respectively. Multivariate analysis revealed that $6 \mathrm{MWD}$ and the FBS score were significant predictors of HRQL.

Conclusions: Our findings suggest that 6MWD and the FBS score are useful parameters for evaluating HRQL in patients with pMAC. Further studies should investigate the impact of 6WMT parameters on disease progression, treatment responses, and prognosis.

Keywords: Nontuberculous mycobacteria, Mycobacterium avium complex, Six-minute walk test, Health-related quality of life, 36-item short form health survey, St. George's respiratory questionnaire

\footnotetext{
* Correspondence: n-hasegawa@z8.keio.jp

${ }^{3}$ Center for Infectious Diseases and Infection Control, Keio University School

of Medicine, 35 Shinanomachi Shinjuku, Tokyo 160-8582, Japan

Full list of author information is available at the end of the article
}

(c) The Author(s). 2018 Open Access This article is distributed under the terms of the Creative Commons Attribution 4.0 International License (http://creativecommons.org/licenses/by/4.0/), which permits unrestricted use, distribution, and reproduction in any medium, provided you give appropriate credit to the original author(s) and the source, provide a link to the Creative Commons license, and indicate if changes were made. The Creative Commons Public Domain Dedication waiver (http://creativecommons.org/publicdomain/zero/1.0/) applies to the data made available in this article, unless otherwise stated. 


\section{Background}

The increasing prevalence of pulmonary infections due to nontuberculous mycobacteria (NTM) is an emerging public health concern worldwide [1, 2]. Pulmonary Mycobacterium avium complex (pMAC) disease, the most common form of NTM infection, presents as a chronic, slowly progressive disease in immunocompetent patients [3]. It causes chronic pulmonary diseases such as asthma, chronic obstructive pulmonary disease (COPD), and interstitial lung disease (ILD) and is generally incurable, requires long-term antimicrobial therapy, and has a high recurrence rate after treatment discontinuation [3]. Because of the increasing chronicity of pMAC disease, patient-reported outcome measures that represent the health-related quality of life (HRQL) have become increasingly important to monitor the overall health status of affected patients [4]. It has been reported that HRQL, particularly the physical component, is impaired in patients with pMAC [5].

The six-minute walk test (6MWT) has become a useful tool for assessing the functional status and predicting the prognosis of patients with various pulmonary diseases, including COPD, ILD, sarcoidosis, and primary pulmonary hypertension [6-9]. It is also used as a standardized exercise test for the assessment of lung disease because of its simplicity, low cost, non-invasiveness, ease of performance, and reproducibility $[10,11]$. Recently, a randomized study of inhaled liposomal amikacin revealed an improvement in 6MWT parameters in patients with pNTM disease [12]. With regard to the clinical significance of $6 \mathrm{MWT}$ for pMAC disease, only one study has assessed the relationship between the six-minute walk distance (6MWD) and HRQL evaluated using St. George's Respiratory Questionnaire (SGRQ) [13]. However, the correlations between HRQL and various 6MWT parameters remain unknown.

The aim of the present study was to investigate the relationship of $6 \mathrm{MWT}$ parameters with clinical parameters, including pulmonary function test (PFT) findings, and HRQL. The tested hypothesis was that $6 \mathrm{MWT}$ parameters could be useful for evaluating HRQL in patients with pMAC disease.

\section{Methods}

\section{Study design and study population}

This cross-sectional study included adult patients with pMAC who were recruited from the prospective, observational cohort registry at Keio University Hospital (UMIN000007546). PMAC was diagnosed on the basis of statements published by the American Thoracic Society (ATS) and Infectious Disease Society of America in 2007 [3]. The Keio University Hospital institutional review board approved the study protocol (\# 20110267), and written informed consent was obtained from each patient. All patients with pMAC who participated in 6MWT and completed questionnaires assessing HRQL between May 2012 and November 2013 were included.

\section{MWT}

All patients were instructed to walk in a hallway for 6 min according to ATS guidelines [10]. Oxygen saturation by pulse oximetry $\left(\mathrm{SpO}_{2}\right)$ and heart rate $(\mathrm{HR})$ were monitored throughout the 6-min walk. The total distance walked and the oxygen saturation and HR (per minute) measured from start to finish were recorded. The patients were assessed using the Borg Dyspnea Scale at the end of the test [14]. Other parameters such as the distance-saturation product (DSP) [6] and desaturation area (DA) [15] were also calculated.

\section{HRQL assessments}

For the evaluation of HRQL, all patients completed the 36-Item Short Form Health Survey (SF-36), version 2 [16] and SGRQ in Japanese [17]. Both these questionnaires have been validated for use in patients with pMAC disease [5]. The SF-36 comprises physical, mental, and role/social domains, and the respective summary scores were adjusted for Japanese patients [18] before analysis. All scores were transformed to fit a norm-based score of 50 and standard deviation of 10, and lower scores indicated a poorer health status. With regard to SGRQ (range, 0-100), we calculated the total score as well as scores for Symptoms, Activity, and Impacts domains, which evaluate respiratory symptoms, physical activity impairment, and social and psychological disturbances, respectively. Lower scores indicated a better health status.

\section{Assessment of clinical parameters}

Clinical parameters, including sex, age at diagnosis, disease duration, body mass index (BMI), smoking status, underlying pulmonary diseases, comorbidities assessed using the age-adjusted Charlson comorbidity index (CCI) [19], and sputum smear and culture findings for MAC within the previous year were recorded at the time of enrolment. PFT was performed using an electronic spirometer (Chestac-9800 or HI-801; Chest M.I., Tokyo, Japan) when the patient was in a stable condition after study enrolment. MAC isolates were identified as previously described [20]. High-resolution computed tomography (HRCT) images were evaluated for cavitary lesions and the radiological pattern: nodular/ bronchiectatic (NB), fibrocavitary (FC), NB + FC, and unclassified [21].

\section{Statistical analysis}

Correlations between two continuous variables were analyzed using Spearman's correlation coefficients. 
Comparisons between two groups were conducted using the Wilcoxon rank sum test. To identify 6MWT parameters that predicted HRQL, parameters that showed a significant association with SGRQ and SF-36 scores in univariate analysis were entered into a stepwise forward and backward multiple regression model for multivariate analysis. All $P$-values were two-tailed, and a value of $<0.05$ was considered statistically significant. All statistical analyses were conducted using JMP v11.0 (SAS Institute Japan Ltd., Tokyo, Japan).

Table 1 Clinical characteristics of patients with pulmonary Mycobacterium avium complex disease $(n=103)$

\begin{tabular}{|c|c|}
\hline \multicolumn{2}{|l|}{ Variable } \\
\hline Age, years & $68(64-75)$ \\
\hline Sex, Male/Female & $23(22) / 80(78)$ \\
\hline Disease duration, years & $5.8(2.3-10.1)$ \\
\hline $\mathrm{BMl}, \mathrm{kg} / \mathrm{m}^{2}$ & $19.2(17.5-20.4)$ \\
\hline \multicolumn{2}{|l|}{ Smoking status } \\
\hline Never/ Former/ Current & $92(89) / 11(11) / 0(0)$ \\
\hline Charlson comorbidity index & $4(4-5)$ \\
\hline \multicolumn{2}{|l|}{ Underlying pulmonary diseases } \\
\hline Old pulmonary tuberculosis & $11(11)$ \\
\hline Bronchial asthma & $4(4)$ \\
\hline Lung cancer & $1(1)$ \\
\hline \multicolumn{2}{|c|}{ Sputum findings for NTM infection within the previous year } \\
\hline Smear/culture positivity & $31(30) / 62(60)$ \\
\hline$\% F V C, \%$ & $94(80-107)$ \\
\hline$\% \mathrm{FEV}_{1}, \%$ & $87(73-98)$ \\
\hline $\mathrm{FEV}_{1} / \mathrm{FVC}<70 \%$ & $35(34)$ \\
\hline$\% \mathrm{FEV}_{1}<80 \%$ & $39(38)$ \\
\hline Presence of cavitary lesions & $31(30.1)$ \\
\hline \multicolumn{2}{|l|}{ Radiological pattern } \\
\hline NB/FC/NB + FC/unclassified & $81(78.6) / 3(2.9) / 15(14.5) / 4(3.9)$ \\
\hline \multicolumn{2}{|l|}{ SF-36 scores } \\
\hline PCS & $48(38-54)$ \\
\hline MCS & $51(42-56)$ \\
\hline RCS & $48(43-54)$ \\
\hline \multicolumn{2}{|l|}{ SGRQ scores } \\
\hline Symptoms & $31(15-48)$ \\
\hline Activity & $24(6-48)$ \\
\hline Impacts & 9 (3-29) \\
\hline Total & $19(9-36)$ \\
\hline
\end{tabular}

Data are shown as number (\%) of patients or medians (interquartile ranges) $B M I$ body mass index, $F C$ fibrocavitary, $F V C$ forced volume capacity, $F E V$, forced expiratory volume in $1 \mathrm{~s}, N B$ nodular/bronchiectatic, MCS mental component summary, NTM nontuberculous mycobacteria, PCS physical component summary, RCS role/social component summary, SF-36 36-Item Short Form Health Survey, SGRQ St. George's Respiratory Questionnaire

\section{Results}

Patient characteristics and SF-36 and SGRQ scores

In total, 103 patients with pMAC were enrolled. Table 1 shows the clinical characteristics of the patients. The median [interquartile range (IQR)] age of patients was 68 (64-75) years. Eighty (78\%) patients were women and $92(89 \%)$ patients were never-smokers. Underlying pulmonary diseases were present in $16(15 \%)$ patients. The median PFT values were within the normal range. On HRCT, 31 (30.1\%) patients showed cavitary lesions, and the NB pattern was most commonly observed (81 patients, $78.6 \%$ ). With regard to HRQL, the median scores for the physical and role/social domains in SF-36 were decreased, whereas the median Symptoms, Activity, and total scores in SGRQ were increased.

\section{MWT parameters}

Table 2 shows the findings of 6WMT for the $103 \mathrm{pa}$ tients. The median (IQR) 6MWD)was 410 (365-450) m. The median (IQR) initial and lowest $\mathrm{SpO}_{2}$ values were 96\% (96-97\%) and 94\% (92-95\%), respectively, while the median (IQR) initial and final HR values were 76 (67$85)$ and 106 (97-116) beats/minute, respectively. The median (IQR) final Borg scale (FBS) score, DSP, and DA were $0.5(0.5-2), 385(338-423) \mathrm{m} \%$, and 32 (27-40) units.

\section{Correlations among 6MWT parameters and clinical parameters}

Table 3 shows Spearman's correlations among 6MWT parameters and clinical parameters for the 103 patients. 6MWD showed a strong correlation with DSP, DA, and the lowest $\mathrm{SpO}_{2}$. The initial $\mathrm{SpO}_{2}$ was moderately correlated with the lowest $\mathrm{SpO}_{2}$ or $\mathrm{DA}$ and the initial and final HRs. Age was significantly correlated with $6 \mathrm{MWD}$, the FBS score, and DSP. Disease duration and age-adjusted CCI were also weakly correlated with the FBS score. Finally, the percentage functional volume

Table 2 Results of the six-minute walk test for patients with pulmonary Mycobacterium avium complex disease $(n=103)$

\begin{tabular}{ll}
\hline Variables & \\
\hline $6 \mathrm{MWD}, \mathrm{m}$ & $410(365-450)$ \\
Initial SpO & $\%$ \\
Lowest $\mathrm{SpO}_{2}, \%$ & $96(96-97)$ \\
Initial heart rate, beats/minute & $94(92-95)$ \\
Final heart rate, beats/minute & $76(67-85)$ \\
Final Borg scale score & $106(97-116)$ \\
DSP, m\% & $0.5(0.5-2)$ \\
DA, units & $385(338-423)$ \\
\hline
\end{tabular}

Data are shown as medians (interquartile ranges) 6MWD six-minute walk distance, $D A$ desaturation area, DSP distance-saturation product, $\mathrm{SpO}_{2}$ oxygen saturation by pulse oximetry 
Table 3 Spearman's correlations among six-minute walk test parameters and clinical parameters for patients with pulmonary Mycobacterium avium complex disease $(n=103)$

\begin{tabular}{|c|c|c|c|c|c|c|c|c|c|c|c|c|c|}
\hline & Age & $\begin{array}{l}\text { Disease } \\
\text { duration }\end{array}$ & $\mathrm{BMI}$ & $\mathrm{CCl}$ & $\% F V C$ & $\% \mathrm{FEV}_{1}$ & $6 \mathrm{MWD}$ & $\begin{array}{l}\text { Initial } \\
\mathrm{SpO}_{2} \\
\end{array}$ & $\begin{array}{l}\text { Lowest } \\
\mathrm{SpO}_{2}\end{array}$ & $\begin{array}{l}\text { Initial } \\
\text { HR }\end{array}$ & $\begin{array}{l}\text { Final } \\
\text { HR }\end{array}$ & FBS & DSP \\
\hline \multicolumn{14}{|l|}{ Age } \\
\hline $\begin{array}{l}\text { Disease } \\
\text { duration }\end{array}$ & -0.09 & & & & & & & & & & & & \\
\hline $\mathrm{BMI}$ & 0.01 & 0.10 & & & & & & & & & & & \\
\hline $\mathrm{CCl}$ & $0.32^{\ddagger}$ & -0.04 & 0.09 & & & & & & & & & & \\
\hline$\% F V C$ & -0.06 & -0.08 & $0.21^{*}$ & $\begin{array}{l}- \\
0.05\end{array}$ & & & & & & & & & \\
\hline$\% \mathrm{FEV}_{1}$ & 0.05 & -0.19 & 0.02 & $\overline{-} 0.01$ & $0.78^{\S}$ & & & & & & & & \\
\hline $6 \mathrm{MWD}$ & $-0.34^{\ddagger}$ & -0.03 & -0.16 & $\begin{array}{l}- \\
0.16\end{array}$ & 0.18 & $0.21^{*}$ & & & & & & & \\
\hline Initial SpO2 & -0.03 & -0.18 & -0.12 & -0.05 & $0.21^{*}$ & $0.32^{\ddagger}$ & 0.08 & & & & & & \\
\hline Lowest SpO2 & -0.07 & -0.11 & -0.05 & $\begin{array}{l}- \\
0.08\end{array}$ & $0.23^{*}$ & $0.27^{\dagger}$ & 0.03 & $0.44^{\S}$ & & & & & \\
\hline Initial HR & -0.06 & 0.06 & $\overline{0.24^{*}}$ & $\begin{array}{l}- \\
0.11\end{array}$ & -0.13 & -0.05 & -0.01 & -0.17 & -0.17 & & & & \\
\hline Final HR & -0.16 & -0.04 & -0.07 & -0.13 & -0.18 & -0.11 & 0.18 & -0.17 & -0.17 & $0.56^{\S}$ & & & \\
\hline FBS & $0.28^{\dagger}$ & $0.23^{*}$ & -0.09 & $0.28^{\dagger}$ & $-0.22^{*}$ & -0.12 & -0.17 & -0.05 & -0.08 & -0.002 & -0.04 & & \\
\hline DSP & $-0.35^{\ddagger}$ & -0.05 & -0.15 & -0.17 & $0.23^{*}$ & $0.26^{+}$ & $0.99^{\S}$ & 0.14 & 0.16 & -0.03 & 0.16 & -0.17 & \\
\hline DA & 0.06 & 0.17 & 0.07 & 0.08 & $-0.25^{*}$ & $\overline{-} .29^{\dagger}$ & -0.01 & $-0.54^{\S}$ & $-0.95^{\S}$ & $0.20^{*}$ & $0.24^{*}$ & 0.11 & -0.14 \\
\hline
\end{tabular}

6MWD six-minute walk distance, $6 M W T$ six-minute walk test, $B M I$ body mass index, $B S$ Borg scale, $C C l$ Charlson comorbidity index, $D A$ Desaturation area, DSP Distance-saturation product, $F B S$ final Borg scale, $F E V$, forced expiratory volume in $1 \mathrm{~s}$, FVC forced volume capacity, $H R$ heart rate, $S p O_{2}$ oxygen saturation by pulse oximetry

${ }^{*} P<0.05,{ }^{\dagger} P<0.01,{ }^{\ddagger} P<0.001,{ }^{\S} P<0.0001$

capacity (\%FVC) and percentage forced expiratory volume in $1 \mathrm{~s}\left(\% \mathrm{FEV}_{1}\right)$ showed significant but weak correlations with the initial and lowest $\mathrm{SpO}_{2}$ values, DSP, and DA.

\section{Correlations among 6MWT parameters or clinical parameters and SF-36 and SGRQ scores}

Table 4 shows the correlations among 6MWT parameters or clinical parameters and SF-36 and SGRQ scores for the 103 patients. The physical component summary (PCS) score in SF-36 was strongly correlated with the Activity and total scores in SGRQ. The mental component summary (MCS) and role/social component summary (RCS) scores were also significantly correlated with the SGRQ scores, although the correlations were weaker than that of the PCS score. All SGRQ scores showed a significant negative correlation with 6MWD $[\rho=(-0.54)-(-0.32)]$ and DSP $[\rho=(-0.29)-(-0.55)]$ and a significant positive correlation with the FBS score $(\rho=0.34-0.58)$. The PCS and RCS scores in SF-36 showed a significant positive correlation with 6MWD $(\rho=0.39$ and $\rho=0.20$, respectively) and a significant negative correlation with the FBS score
( $\rho=-0.50$ and $\rho=-0.24$, respectively). The PCS score also showed a significant positive correlation with DSP $(\rho=0.40)$. Regarding the correlations among clinical parameters and SF-36 and SGRQ scores, all SGRQ scores showed a significant negative correlation with \%FVC $[\rho=(-0.43)-(-0.28)]$ and $\% \mathrm{FEV}_{1}[\rho=(-0.35)-(-0.26)]$. The Activity, Impacts, and Total scores in SGRQ showed significant positive correlation $(\rho=0.22-0.45)$ and the PCS score in SF-36 showed significant negative correlation $(\rho=-0.51)$ with age. Moreover, the Symptoms, Impacts, and Total scores in SGRQ showed significant negative correlation $[\rho=(-0.22)-(-0.30)]$ and the RCS score in SF-36 showed significant positive correlation $(\rho=0.20)$ with BMI. The Activity score in SGRQ showed significant positive correlation $(\rho=0.33)$ and the PCS score in SF-36 showed significant negative correlation $(\rho=-0.35)$ with age-adjusted CCI. We also analyzed the data for the never smoker group $(n=92)$ alone in Additional file 1: Table S4. Correlations among 6MWT parameters or clinical parameters and SF-36 and SGRQ scores were the same as that for all patients including former-smoker patients $(n=103)$. 
Table 4 Spearman's correlations among six-minute walk test parameters or clinical parameters and 36-Item Short Form Health Survey and St George's Respiratory Questionnaire scores for patients with pulmonary Mycobacterium avium complex disease $(n=$ 103)

\begin{tabular}{|c|c|c|c|c|c|c|c|c|}
\hline & & \multicolumn{4}{|l|}{ SGRQ } & \multicolumn{3}{|l|}{ SF-36 } \\
\hline & & Symptoms & Activity & Impacts & Total & $\overline{P C S}$ & MCS & RCS \\
\hline \multirow[t]{4}{*}{ SGRQ } & Symptoms & & & & & & & \\
\hline & Activity & $0.51^{\S}$ & & & & & & \\
\hline & Impacts & $0.68^{\S}$ & $0.67^{\S}$ & & & & & \\
\hline & Total & $0.77^{\S}$ & $0.88^{\S}$ & $0.91^{\S}$ & & & & \\
\hline \multirow[t]{3}{*}{ SF-36 } & PCS & $-0.44^{\S}$ & $-0.73^{\S}$ & $-0.60^{\S}$ & $-0.70^{\S}$ & & & \\
\hline & MCS & $-0.32^{\ddagger}$ & $-0.27^{\dagger}$ & $-0.28^{\dagger}$ & $-0.29^{\dagger}$ & 0.08 & & \\
\hline & RCS & $-0.28^{\dagger}$ & $-0.42^{\S}$ & $-0.40^{\S}$ & $-0.44^{\S}$ & $0.25^{*}$ & 0.15 & \\
\hline \multirow[t]{8}{*}{ 6WMT } & $6 \mathrm{MWD}$ & $-0.27^{\dagger}$ & $-0.54^{\S}$ & $-0.32^{\dagger}$ & $-0.44^{\S}$ & $0.39^{\S}$ & 0.08 & $0.20^{*}$ \\
\hline & Initial $\mathrm{SpO}_{2}$ & 0.0002 & -0.12 & -0.08 & -0.09 & 0.05 & -0.15 & -0.09 \\
\hline & Lowest $\mathrm{SpO}_{2}$ & -0.15 & -0.14 & -0.21 & -0.19 & 0.10 & -0.01 & -0.05 \\
\hline & Initial HR & 0.16 & 0.03 & 0.15 & 0.11 & -0.09 & 0.03 & -0.06 \\
\hline & Final HR & 0.11 & -0.04 & 0.04 & 0.03 & 0.07 & 0.00 & -0.02 \\
\hline & FBS & $0.34^{\ddagger}$ & $0.56^{\S}$ & $0.54^{\S}$ & $0.58^{\S}$ & $-0.50^{\S}$ & -0.17 & $-0.24^{*}$ \\
\hline & DSP & $-0.29^{+}$ & $-0.55^{\S}$ & $-0.33^{\ddagger}$ & $-0.46^{\S}$ & $0.40^{\S}$ & 0.06 & 0.19 \\
\hline & DA & 0.14 & 0.12 & 0.19 & 0.17 & -0.09 & 0.04 & 0.07 \\
\hline \multirow[t]{6}{*}{ Clinical parameters } & Age & 0.09 & $0.45^{\S}$ & $0.22^{*}$ & $0.36^{\ddagger}$ & $-0.51^{\S}$ & 0.10 & -0.18 \\
\hline & Disease duration & 0.16 & 0.13 & 0.11 & 0.18 & -0.06 & -0.02 & -0.01 \\
\hline & BMI & $-0.30^{+}$ & -0.08 & $-0.27^{\dagger}$ & $-0.22^{*}$ & 0.14 & 0.07 & $0.20^{*}$ \\
\hline & $\mathrm{CCl}$ & -0.09 & $0.33^{\ddagger}$ & 0.13 & 0.19 & $-0.35^{\ddagger}$ & 0.04 & -0.05 \\
\hline & $\% F V C$ & $-0.41^{\S}$ & $-0.28^{\dagger}$ & $-0.43^{\S}$ & $-0.43^{\S}$ & 0.15 & -0.03 & 0.07 \\
\hline & $\% \mathrm{FEV}_{1}$ & $-0.34^{\ddagger}$ & $-0.26^{\dagger}$ & $-0.32^{\ddagger}$ & $-0.35^{\ddagger}$ & 0.07 & -0.03 & -0.003 \\
\hline
\end{tabular}

6MWD six-minute walk distance, $6 M W T$ six-minute walk test, $B M I$ body mass index, $C C l$ Charlson comorbidity index, DA Desaturation area, $D S P$ Distance-saturation product, FBS final Borg scale, FEV 1 forced expiratory volume in $1 \mathrm{~s}, F V C$ forced volume capacity, MCS mental component summary, PCS physical component summary, RCS role/social component score, SF-36 36-Item Short Form Health Survey, SGRQ St. George's Respiratory Questionnaire, SpO ${ }_{2}$ oxygen saturation by pulse oximetry

${ }^{*} P<0.05,{ }^{\dagger} P<0.01,{ }^{\ddagger} P<0.001,{ }^{\S} P<0.0001$

\section{Comparisons of 6MWT parameters and questionnaire} scores between patients with cavitary lesions and those without

We also evaluated whether 6MWT parameters and the questionnaire scores differed between patients with cavitary lesions $(n=31,30.1 \%)$ on HRCT and those without ( $n=72,69.9 \%$; Additional file 1: Table S6), because cavitary lesions have been reported as a prognostic factor for pMAC disease [22, 23]. While $6 \mathrm{MWT}$ parameters except the initial HR showed no significant differences between the two groups, the Symptoms and Impacts scores in SGRQ were significantly higher in patients with cavitary lesions than in those without.

\section{Multivariate analysis for predictors of SGRQ and SF-36 scores}

We performed stepwise multiple regression analysis to determine the association of SGRQ and SF-36 scores with age, sex, age-adjusted CCI, BMI, smoking status, disease duration, underlying pulmonary diseases, positive sputum smear or culture findings, presence of cavitary lesions, pulmonary function, 6MWD, DSP, and the FBS score (Table 5). The MCS and RCS scores were excluded because they only showed weak correlations with 6WMT parameters. We chose 6MWT parameters and clinical parameters for stepwise multiple regression analysis that showed significant correlations with SGRQ or SF-36 scores as shown in Table 4 or that were thought to be clinically important from previous studies. \%FVC, sex, and BMI were found to be significant predictors of the Symptoms score in SGRQ, accounting for $21.2 \%$ variance, while DSP, the FBS score, and BMI were found to be significant predictors of the Activity score in SGRQ, accounting for $54.0 \%$ variance. The FBS score, \%FVC, 6MWD, and BMI were found to be significant predictors of the Impacts score in SGRQ, accounting for $38.9 \%$ variance. The FBS score, 6MWD, BMI, and \%FVC were found to be significant predictors of the SGRQ total score, accounting for $49.4 \%$ variance. Finally, the 
Table 5 Multivariate analysis for predictors of 36-Item Short Form Health Survey and St George's Respiratory Questionnaire scores for patients with pulmonary Mycobacterium avium complex disease $(n=103)$

\begin{tabular}{|c|c|c|c|}
\hline $\mathrm{HRQL}$ & Determinants & $P$-value & Cumulative $R^{2}, \%$ \\
\hline \multicolumn{4}{|l|}{ SGRQ scores } \\
\hline \multirow[t]{3}{*}{ Symptoms } & $\% F V C$ & 0.0002 & 14.6 \\
\hline & Sex & 0.0076 & 21.2 \\
\hline & BMI & 0.0303 & 27.9 \\
\hline \multirow[t]{3}{*}{ Activity } & DSP & $<0.0001$ & 33.5 \\
\hline & FBS & $<0.0001$ & 51.3 \\
\hline & BMI & 0.0248 & 54.0 \\
\hline \multirow[t]{4}{*}{ Impacts } & FBS & $<0.0001$ & 20.6 \\
\hline & $\% F V C$ & 0.0020 & 28.7 \\
\hline & $6 \mathrm{MWD}$ & 0.0192 & 33.0 \\
\hline & BMl & 0.0048 & 38.9 \\
\hline \multirow[t]{4}{*}{ Total } & FBS & $<0.0001$ & 24.3 \\
\hline & $6 \mathrm{MWD}$ & $<0.0001$ & 39.2 \\
\hline & BMl & 0.0005 & 47.0 \\
\hline & $\% F V C$ & 0.0483 & 49.4 \\
\hline \multicolumn{4}{|l|}{ SF-36 } \\
\hline \multirow[t]{5}{*}{ PCS } & FBS & $<0.0001$ & 22.4 \\
\hline & $6 \mathrm{MWD}$ & $<0.0001$ & 36.2 \\
\hline & Age & 0.0014 & 43.2 \\
\hline & Sex & 0.0179 & 46.8 \\
\hline & Disease duration & 0.0462 & 49.2 \\
\hline
\end{tabular}

6MWD six-minute walk distance, $6 M W T$ six-minute walk test, $B M I$ body mass index, $D A$ desaturation area, DSP distance-saturation product, $F E V_{1}$ forced expiratory volume in $1 \mathrm{~s}, F B S$ final Borg scale, FVC forced volume capacity, $P C S$ physical component summary, SGRQ St. George's Respiratory Questionnaire, SF-36 36-Item Short Form Health Survey, $\mathrm{SpO}_{2}$ oxygen saturation by pulse oximetry

FBS score, 6MWD, age, sex, and disease duration were found to be significant predictors of the physical component summary (PCS) score in SF-36, accounting for $49.2 \%$ variance. We also analyzed the data for the never smoker group $(n=92)$ alone in Additional file 1: Table S5. Correlations among 6MWT parameters and SF-36 and SGRQ scores were the same as that of all patients including former-smoker patients $(n=103)$. Hence, it was concluded that $6 \mathrm{MWD}$ and the FBS score are useful $6 \mathrm{MWT}$ parameters for the prediction of HRQL in patients with pMAC disease in all patients, including the never smoker group $(n=92)$ and former-smoker patients $(n=103)$ (Table 5).

\section{Discussion}

In the present study, we evaluated the relationship of $6 \mathrm{MWT}$ parameters with clinical parameters, including PFT findings, and HRQL in patients with pMAC disease. The findings revealed that $6 \mathrm{MWD}$ and the FBS score showed strong correlations with the PCS score in SF-36 and all SGRQ scores except for the Symptoms score. Furthermore, \%FVC and BMI, which weakly correlated with 6MWD and the FBS score, were important predictors of the SGRQ scores.

The relationship between 6MWD, which is classically used as the primary outcome of 6MWT, and HRQL assessed using different tools (including SGRQ and SF-36) has been determined in patients with various chronic pulmonary diseases [24, 25], and it was found to correlate negatively with the total SGRQ score in patients with COPD and ILD [26-28]. A previous study on pMAC disease reported that 6WMD significantly correlated with the Activity $(\rho=-0.45)$ and total $(\rho=-0.31)$ scores in SGRQ [13], while in another study, 6MWD showed a significant positive correlation with the total SF-36 score $(\rho=0.37)$ in patients with sarcoidosis [29]. Consistent with findings in previous studies, our data demonstrated that 6MWD was significantly correlated with SGRQ scores and the PCS score in SF-36 and was one of the important predictors of HRQL according to multivariate analysis.

In addition to 6WMD, we found that the FBS score was also an important predictor of SGRQ scores and the PCS score in SF-36. Dyspnea reflects the physiology of exercise limitation as well as the impact of exercise limitation on daily life [30]. In addition, scores for dyspnea assessed using the Borg scale exhibit good reliability and are used as a marker of patient-reported fatigue that reflects a local muscle phenomenon as well as general fatigue in patients with chronic pulmonary diseases [14]. Previous studies reported a significant correlation between the FBS score and all SGRQ scores $(\rho=0.33-0.56)$ in patients with COPD [27] and the total SF-36 score $(\rho=0.45)$ in patients with sarcoidosis [29].

Our multivariate analysis revealed that both 6MWD and the FBS score were important predictors of HRQL parameters. 6MWD is an objective parameter showing significant correlations with measures of peak work capacity in cardiopulmonary exercise tests [24]. Furthermore, a study on COPD revealed that 6MWD was associated with not only lung function but also lower limb strength, including quadriceps strength and the lean leg mass [31]. On the other hand, the Borg scale is a subjective parameter reflecting subjective breathlessness, perceived exertion, and fatigue [14, 24, 32]. A previous study on severe COPD and asthma indicated that the Borg scale could not be reliably predicted by desaturation and PFT findings [32]. In fact, a study on COPD indicated that both 6MWD and the FBS score were significant independent prognostic factors [33]. Thus, 6MWD reflects objective exercise tolerance better than does the FBS score, and both are useful parameters for the assessment of patients with pMAC disease. 
Our multivariate analysis also found that \%FVC and BMI, which have been reported as important clinical factors in previous studies, were predictors of SGRQ scores [13, 20, 23], [34, 35]. In a previous study, FVC showed a negative correlation with SGRQ scores [13], consistent with the findings in this study. Moreover, a substantial decline in $\mathrm{FEV}_{1}$ and $\mathrm{FVC}$ was associated with treatment failure in another study [34]. BMI has been reported as a poor prognostic factor for pulmonary NTM infection [20, 23, 35]. Notably, our results indicated partially significant but weak correlations among BMI, 6WMD or the FBS score, and PFT findings. Therefore, \%FVC and BMI, in addition to 6MWD and the FBS score, may be useful parameters for clinical assessments, including HRQL evaluation.

Our study has several potential limitations. First, we may have excluded patients with more severe pMAC disease, considering we only included patients who could complete PFT, 6MWT, and HRQL questionnaires. Second, this was a cross-sectional study; therefore, we could not determine causal associations, particularly with regard to the influence of treatment. Third, our study mainly included female patients, consistent with the participants of previous studies about pMAC disease [1, 35-37]. Comparison between sexes shows significantly lower BMI in women (Additional file 1: Table S7); this may have affected our results, because BMI is a significant predictor of all SGRQ scores (Table 5). Moreover, we could not evaluate the impact of 6MWT parameters on the prognosis. However, a shorter 6MWD is reported to be strongly correlated with an increased risk of mortality in patients with other chronic pulmonary diseases, including COPD, ILD, and pulmonary hypertension [24, 25, 38-40]. Therefore, we believe that $6 \mathrm{MWT}$ may be useful for the assessment of prognosis in patients with pMAC disease.

\section{Conclusions}

Our findings suggest that 6MWD and the FBS score are useful 6MWT parameters for the prediction of HRQL in patients with pMAC disease. However, further studies are needed to investigate the impact of 6WMT parameters on disease progression, treatment responses, and prognosis.

\section{Additional file}

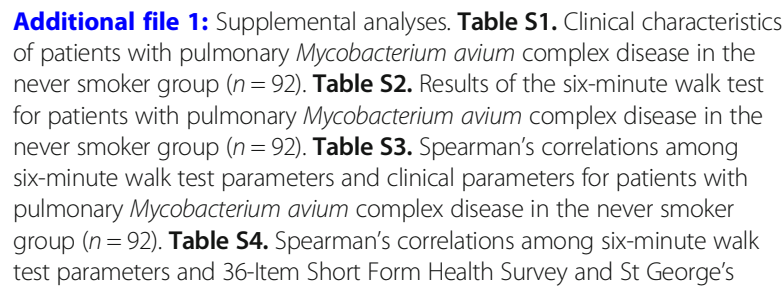

Respiratory Questionnaire scores for patients with pulmonary Mycobacterium avium complex disease in the never smoker group $(n=92)$. Table S5. Multivariate analysis for predictors of 36-Item Short Form Health Survey and St George's Respiratory Questionnaire scores for patients with pulmonary Mycobacterium avium complex disease in the never smoker group $(n=92)$. Table S6. Comparisons of six-minute walk test parameters and 36-Item Short Form Health Survey and St George's Respiratory Questionnaire scores with or without cavitary lesions $(n=103)$. Table S7. Comparisons of clinical characteristics in pulmonary Mycobacterium avium complex disease patients between male and female $(n=103)$. Table S8. Multivariate analysis for predictors of 36-Item Short Form Health Survey and St George's Respiratory Questionnaire scores for patients with pulmonary Mycobacterium avium complex disease in female alone $(n=80)$. Table S9. Comparisons of 36-Item Short Form Health Survey and St George's Respiratory Questionnaire scores with or without several clinical parameters $(n=103)$. (DOCX $55 \mathrm{~kb})$

\section{Abbreviations}

6MWD: six-minute walk distance; 6MWT: six-minute walk test; ATS: American Thoracic Society; BMI: body mass index; CCl: Charlson comorbidity index; COPD: chronic obstructive pulmonary disease; DA: desaturation area; DSP: distance-saturation product; FC: fibrocavitary; $F_{E V}$ : forced expiratory volume in $1 \mathrm{~s}$; FVC: functional volume capacity; HRQL: health-related quality of life; ILD: interstitial lung disease; IQR: interquartile range; MAC: Mycobacterium avium complex; MCS: mental component summary; NB: nodular/bronchiectatic; PCS: physical component summary; PFT: pulmonary function test; RCS: role/ social component summary; SF-36: 36-Item Short Form Health Survey; SGRQ: St. George's Respiratory Questionnaire; $\mathrm{SpO}_{2}$ : oxygen saturation by pulse oximetry

\section{Acknowledgements}

We thank Shoko Takahashi and Chiyomi Uemura for their assistance with collecting data.

\section{Availability of data and materials}

The data will not be shared with participant confidentiality.

\section{Authors' contributions}

$\mathrm{KY}, \mathrm{TA}$, and $\mathrm{NH}$ designed the study; acquired and interpreted data; and wrote, revised, and approved the final manuscript. HN, SS, and MI designed the study; acquired and interpreted data; and revised and approved the final manuscript. TA, SO, TK, YF, HK, TN, and TB acquired and interpreted data and revised and approved the final manuscript.

Ethics approval and consent to participate

The Keio University Hospital ethics review board approved the study protocol (\# 20110267). All patients provided written informed consent.

Consent for publication

Not applicable.

\section{Competing interests}

The authors declare that they have no competing interests.

\section{Publisher's Note}

Springer Nature remains neutral with regard to jurisdictional claims in published maps and institutional affiliations.

\section{Author details}

'Division of Pulmonary Medicine, Department of Medicine, Keio University School of Medicine, Tokyo, Japan. ${ }^{2}$ Keio University Health Center, Tokyo, Japan. ${ }^{3}$ Center for Infectious Diseases and Infection Control, Keio University School of Medicine, 35 Shinanomachi Shinjuku, Tokyo 160-8582, Japan.

Received: 7 April 2018 Accepted: 5 July 2018

Published online: 13 July 2018

References

1. Prevots DR, Marras TK. Epidemiology of human pulmonary infection with nontuberculous mycobacteria: a review. Clin Chest Med. 2015;36(1):13-34. 
2. Namkoong H, Kurashima A, Morimoto K, Hoshino Y, Hasegawa N, Ato M, et al. Epidemiology of pulmonary nontuberculous mycobacterial disease, Japan(1). Emerg Infect Dis. 2016;22(6):1116-7.

3. Griffith DE, Aksamit T, Brown-Elliott BA, Catanzaro A, Daley C, Gordin F, et al. An official ATS/IDSA statement: diagnosis, treatment, and prevention of nontuberculous mycobacterial diseases. Am J Respir Crit Care Med. 2007; 175(4):367-416.

4. Satta G, McHugh TD, Mountford J, Abubakar I, Lipman M. Managing pulmonary nontuberculous mycobacterial infection. Time for a patientcentered approach. Ann Am Thorac Soc. 2014;11(1):117-21.

5. Asakura T, Funatsu Y, Ishii M, Namkoong H, Yagi K, Suzuki S, et al. Healthrelated quality of life is inversely correlated with $\mathrm{C}$-reactive protein and age in Mycobacterium avium complex lung disease: a cross-sectional analysis of 235 patients. Respir Res. 2015;16:145.

6. Lettieri CJ, Nathan SD, Browning RF, Barnett SD, Ahmad S, Shorr AF. The distance-saturation product predicts mortality in idiopathic pulmonary fibrosis. Respir Med. 2006;100(10):1734-41.

7. Miyamoto S, Nagaya N, Satoh T, Kyotani S, Sakamaki F, Fujita M, et al. Clinical correlates and prognostic significance of six-minute walk test in patients with primary pulmonary hypertension. Comparison with cardiopulmonary exercise testing. Am J Respir Crit Care Med. 2000;161(2 Pt 1):487-92

8. Sciurba F, Criner GJ, Lee SM, Mohsenifar Z, Shade D, Slivka W, et al. Six-minute walk distance in chronic obstructive pulmonary disease: reproducibility and effect of walking course layout and length. Am J Respir Crit Care Med. 2003; 167(11):1522-7.

9. Alhamad EH, Shaik SA, Idrees MM, Alanezi MO, Isnani AC. Outcome measures of the 6 minute walk test: relationships with physiologic and computed tomography findings in patients with sarcoidosis. BMC Pulm Med. 2010;10:42

10. American Thoracic Society. ATS statement: guidelines for the six-minute walk test. Am J Respir Crit Care Med. 2002;166(1):111-7.

11. American Thoracic Society. Erratum: ATS statement: guidelines for the six-minute walk test. Am J Respir Crit Care Med. 2016;193(10):1185.

12. Olivier KN, Griffith DE, Eagle G, McGinnis li JP, Micioni L, Liu K, et al. Randomized trial of liposomal amikacin for inhalation in nontuberculous mycobacterial lung disease. Am J Respir Crit Care Med. 2017;195(6):814-23.

13. Maekawa K, Ito Y, Oga T, Hirai T, Kubo T, Fujita K, et al. High-resolution computed tomography and health-related quality of life in Mycobacterium avium complex disease. Int J Tuberc Lung Dis. 2013;17(6):829-35.

14. Borg GA. Psychophysical bases of perceived exertion. Med Sci Sports Exerc 1982;14(5):377-381

15. Flaherty KR, Andrei AC, Murray S, Fraley C, Colby TV, Travis WD, et al. Idiopathic pulmonary fibrosis: prognostic value of changes in physiology and six-minute-walk test. Am J Respir Crit Care Med. 2006;174(7):803-9.

16. Fukuhara S, Bito S, Green J, Hsiao A, Kurokawa K. Translation, adaptation, and validation of the SF-36 health survey for use in Japan. J Clin Epidemiol. 1998:51(11):1037-44

17. Jones PW, Quirk FH, Baveystock CM, Littlejohns P. A self-complete measure of health status for chronic airflow limitation. The St. George's respiratory questionnaire. Am Rev Respir Dis. 1992;145(6):1321-7.

18. Suzukamo Y, Fukuhara S, Green J, Kosinski M, Gandek B, Ware JE. Validation testing of a three-component model of short Form-36 scores. J Clin Epidemiol. 2011;64(3):301-8.

19. Charlson M, Szatrowski TP, Peterson J, Gold J. Validation of a combined comorbidity index. J Clin Epidemiol. 1994;47(11):1245-51.

20. Ueyama M, Asakura T, Morimoto K, Namkoong H, Matsuda S, Osawa T, et al. Pneumothorax associated with nontuberculous mycobacteria: a retrospective study of 69 patients. Medicine (Baltimore). 2016;95(29):e4246.

21. Asakura $T$, Yamada $Y$, Namkoong $H$, Suzuki S, Niijima $Y$, Kamata $H$, et al. Impact of cavity and infiltration on pulmonary function and healthrelated quality of life in pulmonary Mycobacterium avium complex disease: a 3-dimensional computed tomographic analysis. Respir Med. 2017:126:9-16.

22. Gochi M, Takayanagi N, Kanauchi T, Ishiguro T, Yanagisawa T, Sugita Y. Retrospective study of the predictors of mortality and radiographic deterioration in 782 patients with nodular/bronchiectatic Mycobacterium avium complex lung disease. BMJ Open. 2015;5(8):e008058.

23. Asakura T, Hayakawa N, Hasegawa N, Namkoong H, Takeuchi K, Suzuki S, et al. Long-term outcome of pulmonary resection for nontuberculous mycobacterial pulmonary disease. Clin Infect Dis. 2017;65(2):244-51.
24. Singh SJ, Puhan MA, Andrianopoulos V, Hernandes NA, Mitchell KE, Hill CJ, et al. An official systematic review of the European Respiratory Society/ American Thoracic Society: measurement properties of field walking tests in chronic respiratory disease. Eur Respir J. 2014:44(6):1447-78.

25. Holland AE, Spruit MA, Troosters T, Puhan MA, Pepin V, Saey D, et al. An official European Respiratory Society/American Thoracic Society technical standard: field walking tests in chronic respiratory disease. Eur Respir J. 2014; 44(6):1428-46.

26. Brown CD, Benditt JO, Sciurba FC, Lee SM, Criner GJ, Mosenifar Z, et al. Exercise testing in severe emphysema: association with quality of life and lung function. COPD. 2008:5(2):117-24.

27. Katsura H, Yamada K, Wakabayashi R, Kida K. The impact of dyspnoea and leg fatigue during exercise on health-related quality of life in patients with COPD. Respirology. 2005;10(4):485-90.

28. du Bois RM, Weycker D, Albera C, Bradford WZ, Costabel U, Kartashov A, et al. Six-minute-walk test in idiopathic pulmonary fibrosis: test validation and minimal clinically important difference. Am J Respir Crit Care Med. 2011;183(9):1231-7.

29. Bourbonnais JM, Malaisamy S, Dalal BD, Samarakoon PC, Parikh SR, Samavati L. Distance saturation product predicts health-related quality of life among sarcoidosis patients. Health Qual Life Outcomes. 2012;10:67.

30. Callens E, Graba S, Gillet-Juvin K, Essalhi M, Bidaud-Chevalier B, Peiffer C, et al. Measurement of dynamic hyperinflation after a 6-minute walk test in patients with COPD. Chest. 2009;136(6):1466-72.

31. Hillman CM, Heinecke EL, Hii JW, Cecins NM, Jenkins SC, Eastwood PR. Relationship between body composition, peripheral muscle strength and functional exercise capacity in patients with severe chronic obstructive pulmonary disease. Intern Med J. 2012;42(5):578-81.

32. Mak VH, Bugler JR, Roberts CM, Spiro SG. Effect of arterial oxygen desaturation on six minute walk distance, perceived effort, and perceived breathlessness in patients with airflow limitation. Thorax. 1993;48(1):33-8.

33. Golpe R, Perez-de-Llano LA, Mendez-Marote L, Veres-Racamonde A. Prognostic value of walk distance, work, oxygen saturation, and dyspnea during 6-minute walk test in COPD patients. Respir Care. 2013;58(8):1329-34.

34. Park HY, Jeong BH, Chon HR, Jeon K, Daley CL, Koh WJ. Lung function decline according to clinical course in nontuberculous mycobacterial lung disease. Chest. 2016;150(6):1222-32

35. Hayashi M, Takayanagi N, Kanauchi T, Miyahara Y, Yanagisawa T, Sugita Y. Prognostic factors of 634 HIV-negative patients with Mycobacterium avium complex lung disease. Am J Respir Crit Care Med. 2012;185(5):575-83.

36. Morimoto K, Hasegawa N, Izumi K, Namkoong H, Uchimura K, Yoshiyama T, et al. A laboratory-based analysis of nontuberculous mycobacterial lung disease in Japan from 2012 to 2013. Ann Am Thorac Soc. 2017;14(1):49-56.

37. Morimoto K, Iwai K, Uchimura K, Okumura M, Yoshiyama T, Yoshimori K, et al. A steady increase in nontuberculous mycobacteriosis mortality and estimated prevalence in Japan. Ann Am Thorac Soc. 2014;11(1):1-8.

38. Szekely LA, Oelberg DA, Wright C, Johnson DC, Wain J, Trotman-Dickenson $B$, et al. Preoperative predictors of operative morbidity and mortality in COPD patients undergoing bilateral lung volume reduction surgery. Chest. 1997:111(3):550-8.

39. Spruit MA, Polkey MI, Celli B, Edwards LD, Watkins ML, Pinto-Plata V, et al. Predicting outcomes from 6-minute walk distance in chronic obstructive pulmonary disease. J Am Med Dir Assoc. 2012;13(3):291-7.

40. Durheim MT, Smith PJ, Babyak MA, Mabe SK, Martinu T, Welty-Wolf KE, et al. Six-minute-walk distance and accelerometry predict outcomes in chronic obstructive pulmonary disease independent of global initiative for chronic obstructive lung disease 2011 group. Ann Am Thorac Soc. 2015;12(3):349-56. 